\title{
Dinâmica do estrato arbóreo em uma floresta estacional semidecidual em Uberlândia, Minas Gerais, Brasil
}

\author{
Júlio Henrique R. Magalhães', Jamir A. Prado-Junior'1, Vagner S. Vale² \& Ivan Schiavini' \\ 'Universidade Federal de Uberlândia, Instituto de Biologia, Rua Ceará, s/n, CEP 38400-902, Uberlândia, Brasil \\ 2Universidade Estadual de Goiás, Departamento de Engenharia Florestal, Campus Ipameri, \\ Rodovia GO-330 Km 241, CEP 75780-000, Ipameri, Brasil.
}

Recebido em 27.III.2016

Aceito em 20.XI.2017

DOI 10.21826/2446-8231201772309

\begin{abstract}
RESUMO - O presente estudo objetivou avaliar a dinâmica de um fragmento de floresta estacional semidecidual em Uberlândia, região sudeste do Brasil. Para isso foram utilizados dados de dois levantamentos realizados num intervalo temporal de cinco anos (2006 e 2011). As espécies foram divididas em grupos quanto ao estrato vertical (dossel, intermediário e sub-bosque) e grupo sucessional (pioneiras, secundárias iniciais e secundárias tardias). Foram avaliadas as taxas de recrutamento, mortalidade, incremento e decremento para a comunidade e para cada grupo. Observou-se uma redução na densidade arbórea e um aumento de área basal na comunidade, indicando o avanço no estágio sucessional. A riqueza se manteve estável, mas as taxas variaram muito entre os grupos. As maiores taxas de mortalidade e recrutamento ocorreram, predominantemente, para as espécies de sub-bosque e secundárias iniciais. As taxas de dinâmica dos grupos também refletiram as diferentes estratégias de vida.
\end{abstract}

Palavras-chave: estrato vertical, mortalidade, recrutamento, sucessão

ABSTRACT - Dynamics of the arboreal stratum in a semidecidual seasonal forest in Uberlândia, Minas Gerais, Brazil. This study evaluated the dynamics of a seasonal semi-deciduous forest in Uberlândia,southeastern Brazil. For this purpose, data from two surveys conducted over a five-year timeframe (2006 and 2011) were used. Species were divided into groups as vertical strata (canopy, intermediary and understory) and successional groups (pioneer, early secondary and late secondary), and also evaluated for recruitment, mortality, increment and decrement rates. Both community and species groups were evaluated for dynamic rates. There was a reduction in tree density and an increase in basal area for the community, suggesting an advancing in forest succession. Richness remained stable for the community but rates varied greatly among groups. Understory and early secondary species had the higher rates of mortality and recruitment. Dynamic rates also reflected differences in life strategies among groups.

Keywords: vertical strata, mortality, recruitment, succession

\section{INTRODUÇÃO}

A estrutura e a dinâmica de uma floresta são reflexos da complexa interação entre eventos de perturbação e o processo de regeneração natural ocorrendo através do tempo e espaço (Chazdon et al. 2007). Eventos como a queda de galhos e árvores implicam em mudanças nas condições de vida, particularmente na disponibilidade de luz, influenciando o meio físico e, consequentemente, os processos que impulsionam a dinâmica florestal. Tais mudanças se refletem diretamente na morte e recrutamento dos indivíduos, acarretando flutuações na densidade das diferentes espécies (Sheil et al. 2000, Delcamp et al. 2008).

Após distúrbios localizados, são desencadeadas as fases iniciais de sucessão, geralmente caracterizadas pela expansão da biomassa e da densidade de árvores (Oliveira- Filho et al. 1997, Gomes et al. 2003), além da colonização por novas espécies, levando a um acúmulo gradual de espécies ao longo do tempo (Chazdon et al. 2007). Este aumento na riqueza de espécies tende a alcançar um pico, após o qual ocorre a queda, devido à exclusão competitiva (Odum 2001).
Segundo Chazdon (2012), as hipóteses que previam o equilíbrio de florestas ao longo do tempo têm sido descartadas, uma vez que os distúrbios são frequentes, mesmo durante os estágios tardios de sucessão, inexistindo um momento específico em que a floresta alcance um estado de estabilidade ou clímax. Assim, a floresta pode ser vista como um mosaico de manchas em diferentes estágios de maturidade, cujo ciclo se inicia com uma clareira, sendo possível dividir o processo sucessional em três fases: clareira, construção e fase madura (Whitmore 1989).

Nesse contexto, estudos de dinâmica de florestas tornam-se fundamentais, pois fornecem informações para a compreensão da diversidade e complexidade das populações e comunidades (Sheil et al. 2000), permitindo o monitoramento e a previsão dos processos de transformação das comunidades vegetais isoladas (Higuchi et al. 2008). Tais estudos utilizam-se comumente de taxas de mortalidade, recrutamento, e ganho e perda de área basal para as populações e a comunidade, no intuito de identificar padrões de variação estrutural ao longo do tempo. 
Dessa forma, o presente estudo tem como objetivo geral descrever a dinâmica de uma comunidade arbórea, incluindo os diferentes estratos da floresta - sub-bosque, intermediário e dossel, para elucidar qual a contribuição de cada estrato na evolução sucessional da floresta.

\section{MATERIAL E MÉTODOS}

\section{Caracterização da área}

$\mathrm{O}$ estudo foi realizado em um fragmento florestal com cerca de 30 ha denominado "Mata do Glória", localizado na Fazenda Experimental do Glória (1856'57' S, $48^{\circ} 12^{\prime} 14^{\prime \prime} \mathrm{W}, 910 \mathrm{msnm}$ ), pertencente à Universidade Federal de Uberlândia. Circundado por áreas antropizadas, compostas por pastagens e lavouras, o fragmento é composto predominantemente por floresta estacional semidecidual (FES) e um pequeno trecho de floresta de galeria, e situa-se em uma área com declividade suave. Para mais informações sobre a área de estudo, ver Lopes et al. (2011).

O clima regional é classificado como Cwa mesotérmico (sistema de Köppen), caracterizado por verões chuvosos (outubro a março) e invernos secos (abril a setembro) (Alvares et al. 2013). Os valores anuais médios de precipitação e temperatura em Uberlândia entre os anos de 1981 a 2008 foram $1584 \mathrm{~mm}$ e $22,5^{\circ} \mathrm{C}$, respectivamente. A pluviosidade média mensal é de 228,5 mm durante os meses chuvosos, e de 35,5 mm nos meses secos (PradoJunior et al. 2010).

O solo da floresta é do tipo latossolo vermelho-escuro distrófico, com textura argilosa (saturação de bases de $29 \pm 11 \%$ no horizonte A1 e 7,4 $\pm 3 \%$ no A3) e apresenta baixa disponibilidade de $\mathrm{Mg}, \mathrm{K}, \mathrm{N}, \mathrm{P}$ e Ca (Haridasan \& Araújo 2005).

\section{Coleta de dados}

Neste estudo foi realizado um novo levantamento fitossociológico da comunidade arbórea amostrada por Lopes et al. (2011) no ano de 2006. Foram alocadas 25 parcelas permanentes contíguas $(20 \times 20 \mathrm{~m})$, totalizando 1 ha de área amostral. Todos os indivíduos arbóreos com circunferência à altura do peito (CAP $1,30 \mathrm{~m}) \geq 15 \mathrm{~cm}$ foram marcados, identificados em nível de espécie e tiveram seu CAP medido e sua altura estimada (Lopes et al. 2011).

Para o estudo da dinâmica florestal, seguiu-se a mesma metodologia aplicada por Lopes et al. (2011). Os indivíduos foram remedidos, os mortos registrados e os recrutas que atingiram o limite de inclusão amostrados. A classificação das famílias seguiu APG IV (APG 2016) e o nome das espécies foi atualizado de acordo com a Lista de Espécies da Flora do Brasil (Flora do Brasil 2020).

\section{Classificação das espécies em grupos sucessionais}

Foi baseada nos trabalhos de Vale et al. (2009), Dias Neto et al. (2009), Prado Junior et al. (2010), Lopes et al. (2011) e Prado Júnior et al. (2012) realizados em florestas estacionais semideciduais na região, além de observações sobre a ocorrência das espécies em campo. As espécies foram separadas em três categorias sucessionais, de acordo com Budowski (1965) e adaptado por Gandolfi et al. (1995), como: pioneiras $(\mathrm{P})$ - dependentes de luz direta, que não ocorrem no sub-bosque, desenvolvendo-se em clareiras ou nas bordas da floresta; secundárias iniciais (SI) - ocorrendo em condições de sombreamento médio ou luminosidade não muito intensa, em clareiras pequenas, bordas de clareiras grandes e bordas de floresta; secundárias tardias (ST) - desenvolvendo-se no sub-bosque em condições de sombra leve ou densa e aí permanecendo ou crescendo até alcançarem o dossel ou a condição de emergentes.

\section{Classificação das espécies quanto ao estrato vertical}

As espécies amostradas foram classificadas quanto à posição no estrato vertical em espécies de dossel, estrato intermediário e sub-bosque utilizando quartil e mediana das alturas da comunidade e das espécies (Vale et al. 2009). Para as espécies com menos de cinco indivíduos (devido à imprecisão nos cálculos de quartil e mediana das alturas), a classificação foi baseada em outros estudos realizados em diferentes fragmentos de floresta estacional semidecidual da região (Vale et al. 2009, Lopes et al. 2014).

\section{Análises dos dados}

Foram calculadas as taxas anuais de mortalidade, recrutamento, incremento ou decremento em área basal para descrever a dinâmica da comunidade arbórea em cada estrato utilizando-se o modelo de taxas exponenciais anuais proposto por Sheil et al. (1995) e Sheil et al. (2000):

$$
\begin{gathered}
\mathrm{M}=\left(1-\left(\frac{\mathrm{N}_{0}-\mathrm{m}}{\mathrm{N}_{0}}\right)^{\frac{1}{\mathrm{t}}}\right) \cdot 100 \\
\mathrm{R}=\left(1-\left(\frac{1-\mathrm{r}}{\mathrm{N}_{\mathrm{t}}}\right)^{\frac{1}{\mathrm{t}}}\right) \cdot 100 \\
\mathrm{D}=\left(1-\left(\frac{\left(A B_{0}-\left(A B_{\mathrm{m}}+A B_{\mathrm{d}}\right)\right.}{\mathrm{AB} \mathrm{B}_{0}}\right)^{\frac{1}{\mathrm{t}}}\right) \cdot 100 \\
\mathrm{I}=\left(1-\left(\frac{\left(1-\left(A B_{\mathrm{t}}+\mathrm{AB}\right)\right.}{\mathrm{AB}}\right)_{\mathrm{t}}^{\frac{1}{\mathrm{t}}}\right) \cdot 100
\end{gathered}
$$

Onde: $\mathrm{M}=$ taxa de mortalidade anual; $\mathrm{R}=$ taxa de recrutamento anual; $\mathrm{D}=$ taxa de decremento em área basal anual; $\mathrm{I}=$ taxa de incremento em área basal anual; $\mathrm{t}=$ intervalo de tempo entre as amostragens; N0 = número inicial de árvores; $\mathrm{Nt}=$ número final de árvores; $\mathrm{m}=$ número de árvores mortas; $r$ = número de árvores recrutadas; $\mathrm{AB} 0=$ área basal inicial; $\mathrm{ABt}=$ área basal final; $\mathrm{ABm}=$ área basal das árvores mortas; $\mathrm{ABd}=$ perda em área basal; $\mathrm{ABr}=$ área basal de árvores recrutas; e $\mathrm{ABg}=$ ganho em área basal.

Os parâmetros fitossociológicos (densidade, frequência, dominância) foram calculados para cada espécie, conforme Muller-Dombois \& Ellenberg (1974), além do índice de 
diversidade de Shannon (H') para a comunidade, através do programa FITOPAC 2.1 (Shepherd 2009) para os dois períodos amostrados.

A normalidade dos dados foi testada utilizando-se o teste de Kolmogorov-Smirnov. Quando os pressupostos de normalidade e homogeneidade de variâncias não foram atendidos, utilizamos testes não paramétricos correspondentes. Foi utilizado o teste $\mathrm{t}$ de Hutcheson (Brower et al. 1997) para avaliar a diferença na diversidade de Shannon (H') entre as duas amostras. Foi utilizado o teste t pareado para avaliar se existe diferença nos valores de área basal e número de indivíduos entre os dois anos de amostragem para cada estrato (dossel, intermediário e subbosque). Foi realizado o teste de Friedman para medidas repetidas, para avaliar se existe diferença entre as taxas de dinâmicas (mortalidade, recrutamento, decremento e incremento), utilizando as parcelas como amostras, e o teste a posteriori de Bonferroni corrigido para comparação entre os estratos. O mesmo teste foi realizado para as taxas de dinâmica dos grupos sucessionais. O nível de significância utilizado para os testes estatísticos foi de $5 \%(\mathrm{p} \leq 0,05)$.

\section{RESULTADOS E DISCUSSÃO}

\section{Parâmetros estruturais}

Entre 2006 e 2011, houve uma redução significativa de 976 para 934 no número de indivíduos $(\mathrm{t}=2,34, \mathrm{gl}=24$, $\mathrm{p}$ $<0,028)$ e um aumento significativo de 26,33 para 27,61 $\mathrm{m} 2$.ha-1 na área basal da comunidade $(\mathrm{t}=3,54, \mathrm{gl}=24, \mathrm{p}<$ 0,01 ) (Tab. 1). Uma vez que florestas maduras apresentam menores densidades de árvores e maiores valores de área basal, enquanto que aquelas em estágios mais iniciais de sucessão formam grandes adensamentos de árvores com pequenos diâmetros (Parthasarathy 1999, Nunes et al. 2003, Vale et al. 2009), a redução na densidade e o aumento na área basal indicam uma evolução para um estágio sucessional mais avançado. $\mathrm{O}$ mesmo foi também relatado para outros estudos em florestas estacionais semideciduais (Appolinario et al. 2005, Oliveira-Filho et al. 2007, Rodrigues-Souza et al. 2015). Este é um padrão comumente encontrado em florestas tropicais, denominado processo de autodesbaste (Oliveira-Filho et al. 1997, Chagas et al. 2001) e associado à fase de crescimento da floresta em que tende a reduzir o número de indivíduos, principalmente os de pequeno porte, concomitante com o crescimento em espessura de árvores que resistem à competição e acumulam biomassa (Machado \& Oliveira-Filho 2010).

\section{Parâmetros florísticos}

Em ambos os censos, as famílias mais representativas em número de espécies foram Fabaceae, Myrtaceae, Rubiaceae, Annonaceae, Lauraceae, Meliaceae e Moraceae, que juntas representaram mais de $40 \%$ do total de indivíduos e $50 \%$ da área basal da comunidade. A grande representatividade de Myrtaceae, Lauraceae e Meliaceae está relacionada a florestas estacionais semideciduais mais conservadas (Vale et al. 2009, Prado Júnior et al. 2014). Essas são as principais famílias registradas em trabalhos em florestas estacionais semideciduais da região (Vale et al. 2009, Dias Neto et al. 2009, Prado Júnior et al. 2010). Também não foram observadas mudanças significativas no número de espécies, que variou de 89 para $84(\mathrm{t}=1,02, \mathrm{gl}=24, \mathrm{p}=$ 0,327 ), ou nos valores de diversidade de Shannon, de 3,65 a 3,64 ( $\mathrm{t}$ de Hutcheson =0,21, $\mathrm{p}=0,819$ ). A ausência de alterações florísticas mais acentuadas na floresta pode estar relacionada ao período de tempo relativamente curto entre as amostragens. Tais mudanças requerem longos períodos de monitoramento, sobretudo em florestas já em estágios mais avançados de sucessão (Oliveira-Filho et al. 2007).

Após o intervalo de cinco anos, deixaram de ocorrer Casearia sylvestris, Bauhinia rufa, B. ungulata, Cardiopetalum calophyllum, Cupania vernalis, Eugenia florida, Guettarda viburnoides e Nectandra megapotamica), todas classificadas como espécies secundárias iniciais. Por outro lado, em 2011 três novas ocorrências foram registradas, todas com apenas um indivíduo, sendo duas secundárias iniciais (Zanthoxylum rhoifolium e Campomanesia guazumifolia) e uma secundária tardia (Xylopia brasiliensis).

Entre as espécies amostradas em ambos os períodos, Siparuna guianensis foi a que apresentou a densidade mais elevada. Contudo, a redução em um terço no número de indivíduos desta espécie influenciou o padrão de dinâmica observado na comunidade, principalmente em relação à diminuição da densidade arbórea. Com um total de 53 indivíduos mortos, $73 \%$ daqueles no sub-bosque e $38 \%$ do total na comunidade, foi a espécie que apresentou as taxas de dinâmica mais aceleradas. Também apresentou o maior recrutamento (24 indivíduos), o que representou $54 \%$ dos recrutas no sub-bosque e $25 \%$ na comunidade. Trata-se de uma espécie secundária inicial com grande plasticidade ecológica considerada das mais frequentes em florestas semidecíduas (Durigan 2002). De acordo com (Meira-Neto et al. 2005), prefere locais com baixo valor de cobertura, o que pode estar relacionado à diminuição de sua densidade no período analisado, já que a floresta, livre de fatores de perturbação intensos, apresentou indícios de avanço para estágios sucessionais mais maduros e, consequentemente, maior cobertura do dossel.

\section{Dinâmica entre os estratos}

Para a comunidade, as taxas de mortalidade $(3,03 \%$. ano- 1$)$, recrutamento $(2,17 \%$.ano- 1$)$, decremento $(1,31 \%$. ano-1) e incremento (2,24\%.ano-1) ficaram dentro dos padrões encontrados em florestas estacionais sem grandes distúrbios (Oliveira et al. 2014), provavelmente devido à não ocorrência de eventos climáticos severos como tempestades (Laurance et al. 2006), períodos de seca prolongados (Chazdon et al. 2008) e por se tratar de uma área protegida. Entretanto, observou-se que estas taxas foram significativamente diferentes entre os estratos (Tab. 2). O sub-bosque apresentou as maiores taxas de dinâmica para todos os parâmetros analisados, demonstrando elevada substituição de indivíduos e variação na área basal. Já o 
Tabela 1. Espécies amostradas em 2006 e 2011 na floresta estacional semidecidual "Mata do Glória", Uberlândia, MG, apresentadas em ordem de densidade absoluta para os dados de 2006. GS = grupo sucessional; EV = estrato vertical; $\mathrm{P}=$ pioneira; $\mathrm{SI}=$ secundária inicial; $\mathrm{ST}=$ secundária tardia; $\mathrm{S}=$ sub-bosque; $\mathrm{I}=$ estrato intermediário; $\mathrm{D}=$ dossel; $\mathrm{DA}=$ densidade absoluta; $\mathrm{FA}=$ frequência absoluta; $\mathrm{AB}=$ área basal em cm² (=dominância absoluta); $\mathrm{DR}=$ densidade relativa; $\mathrm{FR}=$ frequência relativa; $\mathrm{DoR}=$ dominância relativa. *espécies presentes em apenas um ano de amostragem.

\begin{tabular}{|c|c|c|c|c|c|c|c|c|c|c|c|c|c|c|}
\hline \multirow{2}{*}{$\begin{array}{l}\text { Anos } \\
\text { Espécies }\end{array}$} & \multirow[b]{2}{*}{ GS } & \multirow[b]{2}{*}{$\mathrm{EV}$} & \multicolumn{6}{|c|}{2006} & \multicolumn{6}{|c|}{2011} \\
\hline & & & DA & FA & $\mathrm{AB}$ & DR & FR & DoR & DA & FA & $\mathrm{AB}$ & DR & FR & DoR \\
\hline Siparuna guianensis Aubl. & SI & $\mathrm{S}$ & 95 & 21 & 3197.52 & 9.73 & 3.99 & 1.21 & 66 & 21 & 2493.86 & 7.07 & 4.06 & 0.90 \\
\hline $\begin{array}{l}\text { Protium heptaphyllum } \\
\text { (Aubl.) Marchand }\end{array}$ & ST & $\mathrm{D}$ & 63 & 20 & 16270.33 & 6.45 & 3.80 & 6.18 & 57 & 20 & 16254.39 & 6.10 & 3.87 & 5.89 \\
\hline $\begin{array}{l}\text { Casearia grandiflora } \\
\text { Cambess. }\end{array}$ & SI & $\mathrm{I}$ & 59 & 22 & 2999.33 & 6.05 & 4.18 & 1.14 & 50 & 20 & 2675.02 & 5.35 & 3.87 & 0.97 \\
\hline $\begin{array}{l}\text { Aspidosperma discolor } \\
\text { A.DC. }\end{array}$ & SI & I & 55 & 21 & 7488.28 & 5.64 & 3.99 & 2.84 & 63 & 21 & 9078.30 & 6.75 & 4.06 & 3.29 \\
\hline $\begin{array}{l}\text { Duguetia lanceolata A.St.- } \\
\text { Hil. }\end{array}$ & ST & I & 48 & 19 & 2617.70 & 4.92 & 3.61 & 0.99 & 52 & 20 & 3356.64 & 5.57 & 3.87 & 1.22 \\
\hline Pouteria torta (Mart.) Radlk. & SI & $\mathrm{D}$ & 43 & 21 & 13343.71 & 4.41 & 3.99 & 5.07 & 46 & 21 & 14875.79 & 4.93 & 4.06 & 5.39 \\
\hline $\begin{array}{l}\text { Ocotea corymbosa (Meisn.) } \\
\text { Mez }\end{array}$ & ST & $\mathrm{D}$ & 40 & 16 & 34924.54 & 4.10 & 3.04 & 13.26 & 37 & 15 & 36746.67 & 3.96 & 2.90 & 13.31 \\
\hline Cordia sellowiana Cham. & $\mathrm{SI}$ & $\mathrm{I}$ & 39 & 15 & 9288.48 & 4.00 & 2.85 & 3.53 & 39 & 15 & 9889.55 & 4.18 & 2.90 & 3.58 \\
\hline $\begin{array}{l}\text { Cheiloclinium cognatum } \\
\text { (Miers.) .C.Sm. }\end{array}$ & SI & $\mathrm{S}$ & 36 & 17 & 2907.38 & 3.69 & 3.23 & 1.10 & 38 & 18 & 3339.37 & 4.07 & 3.48 & 1.21 \\
\hline $\begin{array}{l}\text { Cryptocarya aschersoniana } \\
\mathrm{Mez}\end{array}$ & ST & $\mathrm{D}$ & 36 & 15 & 6215.60 & 3.69 & 2.85 & 2.36 & 36 & 15 & 5416.40 & 3.85 & 2.90 & 1.96 \\
\hline Virola sebifera Aubl. & $\mathrm{P}$ & $\mathrm{D}$ & 32 & 18 & 6988.79 & 3.28 & 3.42 & 2.65 & 30 & 15 & 6273.95 & 3.21 & 2.90 & 2.27 \\
\hline $\begin{array}{l}\text { Astronium nelson-rosae } \\
\text { Santin }\end{array}$ & ST & $\mathrm{D}$ & 27 & 17 & 5152.32 & 2.77 & 3.23 & 1.96 & 29 & 18 & 5897.11 & 3.10 & 3.48 & 2.14 \\
\hline Inga vera Willd. & SI & I & 23 & 10 & 3030.79 & 2.36 & 1.90 & 1.15 & 29 & 15 & 3706.52 & 3.10 & 2.90 & 1.34 \\
\hline Machaerium villosum Vogel & ST & $\mathrm{D}$ & 19 & 10 & 10402.90 & 1.95 & 1.90 & 3.95 & 17 & 10 & 10726.45 & 1.82 & 1.93 & 3.88 \\
\hline $\begin{array}{l}\text { Siphoneugena densiflora } \\
\text { O.Berg }\end{array}$ & ST & $\mathrm{S}$ & 18 & 11 & 2075.60 & 1.84 & 2.09 & 0.79 & 19 & 13 & 1724.72 & 2.03 & 2.51 & 0.62 \\
\hline Trichilia pallida $\mathrm{Sw}$. & ST & $\mathrm{S}$ & 18 & 10 & 1182.52 & 1.84 & 1.90 & 0.45 & 18 & 11 & 1166.25 & 1.93 & 2.13 & 0.42 \\
\hline Platycyamus regnellii Benth. & ST & $\mathrm{D}$ & 17 & 10 & 14945.66 & 1.74 & 1.90 & 5.68 & 17 & 10 & 17414.10 & 1.82 & 1.93 & 6.31 \\
\hline $\begin{array}{l}\text { Cordiera sessilis (Vell.) } \\
\text { Kuntze }\end{array}$ & SI & $\mathrm{S}$ & 16 & 11 & 667.20 & 1.64 & 2.09 & 0.25 & 16 & 9 & 690.31 & 1.71 & 1.74 & 0.25 \\
\hline Heisteria ovata Benth. & SI & $\mathrm{S}$ & 16 & 10 & 1271.47 & 1.64 & 1.90 & 0.48 & 17 & 10 & 1453.02 & 1.82 & 1.93 & 0.53 \\
\hline Terminalia glabrescens Mart. & SI & $\mathrm{D}$ & 16 & 11 & 6955.93 & 1.64 & 2.09 & 2.64 & 15 & 11 & 7292.06 & 1.61 & 2.13 & 2.64 \\
\hline $\begin{array}{l}\text { Apuleia leiocarpa (Vogel) } \\
\text { J.F.Macbr. }\end{array}$ & SI & $\mathrm{D}$ & 14 & 10 & 9123.10 & 1.43 & 1.90 & 3.46 & 14 & 11 & 9448.27 & 1.50 & 2.13 & 3.42 \\
\hline $\begin{array}{l}\text { Simira sampaioana (Standl.) } \\
\text { Steyerm. }\end{array}$ & ST & I & 14 & 10 & 935.08 & 1.43 & 1.90 & 0.36 & 15 & 10 & 1253.90 & 1.61 & 1.93 & 0.45 \\
\hline Copaifera langsdorffii Desf. & $\mathrm{ST}$ & $\mathrm{D}$ & 13 & 9 & 17584.45 & 1.33 & 1.71 & 6.68 & 13 & 9 & 18691.85 & 1.39 & 1.74 & 6.77 \\
\hline $\begin{array}{l}\text { Tapirira obtusa (Benth.) } \\
\text { J.D.Mitch. }\end{array}$ & SI & $\mathrm{D}$ & 12 & 12 & 13539.53 & 1.23 & 2.28 & 5.14 & 12 & 11 & 14523.88 & 1.28 & 2.13 & 5.26 \\
\hline Faramea hyacinthina Mart. & SI & I & 11 & 9 & 1229.85 & 1.13 & 1.71 & 0.47 & 11 & 9 & 1365.29 & 1.18 & 1.74 & 0.49 \\
\hline $\begin{array}{l}\text { Hirtella gracilipes (Hook.f.) } \\
\text { Prance }\end{array}$ & SI & I & 11 & 11 & 1052.63 & 1.13 & 2.09 & 0.40 & 12 & 12 & 1181.67 & 1.28 & 2.32 & 0.43 \\
\hline Ocotea spixiana (Nees) Mez & ST & $\mathrm{D}$ & 10 & 8 & 4269.19 & 1.02 & 1.52 & 1.62 & 10 & 8 & 4739.24 & 1.07 & 1.55 & 1.72 \\
\hline $\begin{array}{l}\text { Xylopia aromatica (Lam.) } \\
\text { Mart. }\end{array}$ & $\mathrm{P}$ & I & 10 & 6 & 717.81 & 1.02 & 1.14 & 0.27 & 8 & 5 & 827.49 & 0.86 & 0.97 & 0.30 \\
\hline Annona cacans Warm. & ST & $\mathrm{D}$ & 9 & 8 & 5028.78 & 0.92 & 1.52 & 1.91 & 9 & 8 & 5306.80 & 0.96 & 1.55 & 1.92 \\
\hline Maprounea guianensis Aubl. & SI & $\mathrm{D}$ & 9 & 7 & 2106.48 & 0.92 & 1.33 & 0.80 & 7 & 6 & 1268.76 & 0.75 & 1.16 & 0.46 \\
\hline $\begin{array}{l}\text { Pouteria gardneri (Mart. \& } \\
\text { Miq.) Baehni }\end{array}$ & ST & $\mathrm{D}$ & 9 & 8 & 1920.05 & 0.92 & 1.52 & 0.73 & 9 & 8 & 2051.11 & 0.96 & 1.55 & 0.74 \\
\hline Amaioua guianensis Aubl. & SI & I & 7 & 7 & 511.60 & 0.72 & 1.33 & 0.19 & 5 & 5 & 488.39 & 0.54 & 0.97 & 0.18 \\
\hline Ixora brevifolia Benth. & ST & $\mathrm{I}$ & 7 & 5 & 1060.61 & 0.72 & 0.95 & 0.40 & 8 & 6 & 1198.83 & 0.86 & 1.16 & 0.43 \\
\hline
\end{tabular}


Tabela 1. Continuação

\begin{tabular}{|c|c|c|c|c|c|c|c|c|c|c|c|c|c|c|}
\hline \multirow{2}{*}{$\begin{array}{l}\text { Anos } \\
\text { Espécies }\end{array}$} & \multirow[b]{2}{*}{ GS } & \multirow[b]{2}{*}{$\mathrm{EV}$} & \multicolumn{6}{|c|}{2006} & \multicolumn{6}{|c|}{2011} \\
\hline & & & DA & FA & $\mathrm{AB}$ & DR & FR & DoR & $\mathrm{DA}$ & FA & $\mathrm{AB}$ & DR & FR & DoR \\
\hline Margaritaria nobilis L.f. & SI & $\mathrm{D}$ & 7 & 7 & 1504.13 & 0.72 & 1.33 & 0.57 & 5 & 5 & 1170.98 & 0.54 & 0.97 & 0.42 \\
\hline $\begin{array}{l}\text { Schefflera morototoni } \\
\text { (Aubl.) Maguire }\end{array}$ & $\mathrm{P}$ & $\mathrm{D}$ & 7 & 5 & 4146.90 & 0.72 & 0.95 & 1.57 & 6 & 6 & 4732.71 & 0.64 & 1.16 & 1.71 \\
\hline $\begin{array}{l}\text { Ormosia arborea (Vell.) } \\
\text { Harms }\end{array}$ & SI & $\mathrm{D}$ & 6 & 5 & 687.73 & 0.61 & 0.95 & 0.26 & 6 & 5 & 854.80 & 0.64 & 0.97 & 0.31 \\
\hline Casearia sylvestris Sw. * & SI & $\mathrm{I}$ & 5 & 4 & 387.56 & 0.51 & 0.76 & 0.15 & - & - & - & - & - & - \\
\hline Hymenaea courbaril L. & ST & $\mathrm{D}$ & 5 & 4 & 4616.81 & 0.51 & 0.76 & 1.75 & 5 & 4 & 4932.21 & 0.54 & 0.77 & 1.79 \\
\hline $\begin{array}{l}\text { Cariniana estrellensis } \\
\text { (Raddi) Kuntze }\end{array}$ & ST & $\mathrm{D}$ & 4 & 4 & 10913.97 & 0.41 & 0.76 & 4.14 & 4 & 4 & 10956.31 & 0.43 & 0.77 & 3.97 \\
\hline $\begin{array}{l}\text { Piptocarpha macropoda } \\
\text { (DC.) Baker }\end{array}$ & $\mathrm{P}$ & I & 4 & 3 & 1498.21 & 0.41 & 0.57 & 0.57 & 4 & 3 & 1853.60 & 0.43 & 0.58 & 0.67 \\
\hline Roupala montana Aubl. & ST & I & 4 & 3 & 601.17 & 0.41 & 0.57 & 0.23 & 3 & 2 & 202.84 & 0.32 & 0.39 & 0.07 \\
\hline $\begin{array}{l}\text { Sorocea bonplandii (Baill.) } \\
\text { W.C.Burger et al. }\end{array}$ & SI & $\mathrm{S}$ & 4 & 4 & 240.36 & 0.41 & 0.76 & 0.09 & 4 & 4 & 255.17 & 0.43 & 0.77 & 0.09 \\
\hline $\begin{array}{l}\text { Aegiphila integrifolia (Jacq.) } \\
\text { Moldenke }\end{array}$ & $\mathrm{P}$ & I & 3 & 3 & 233.76 & 0.31 & 0.57 & 0.09 & 3 & 3 & 357.62 & 0.32 & 0.58 & 0.13 \\
\hline Coccoloba mollis Casar. & SI & $\mathrm{I}$ & 3 & 2 & 176.28 & 0.31 & 0.38 & 0.07 & 3 & 2 & 232.07 & 0.32 & 0.39 & 0.08 \\
\hline $\begin{array}{l}\text { Handroanthus serratifolius } \\
\text { (A.H.Gentry) S.Grose }\end{array}$ & ST & $\mathrm{D}$ & 3 & 3 & 134.05 & 0.31 & 0.57 & 0.05 & 3 & 3 & 139.68 & 0.32 & 0.58 & 0.05 \\
\hline Jacaranda macrantha Cham. & $\mathrm{P}$ & $\mathrm{D}$ & 3 & 2 & 1349.02 & 0.31 & 0.38 & 0.51 & 3 & 2 & 1421.11 & 0.32 & 0.39 & 0.51 \\
\hline Matayba guianensis Aubl. & SI & I & 3 & 2 & 403.56 & 0.31 & 0.38 & 0.15 & 2 & 2 & 308.96 & 0.21 & 0.39 & 0.11 \\
\hline $\begin{array}{l}\text { Nectandra membranacea } \\
\text { (Sw.) Griseb. }\end{array}$ & SI & $\mathrm{D}$ & 3 & 3 & 1786.45 & 0.31 & 0.57 & 0.68 & 6 & 5 & 1951.88 & 0.64 & 0.97 & 0.71 \\
\hline $\begin{array}{l}\text { Ouratea castaneifolia (DC.) } \\
\text { Engl. }\end{array}$ & SI & I & 3 & 3 & 574.57 & 0.31 & 0.57 & 0.22 & 3 & 3 & 638.69 & 0.32 & 0.58 & 0.23 \\
\hline Platypodium elegans Vogel & SI & $\mathrm{D}$ & 3 & 2 & 3079.35 & 0.31 & 0.38 & 1.17 & 3 & 2 & 3284.48 & 0.32 & 0.39 & 1.19 \\
\hline Vitex polygama Cham. & SI & $\mathrm{D}$ & 3 & 3 & 1515.71 & 0.31 & 0.57 & 0.58 & 3 & 3 & 1530.27 & 0.32 & 0.58 & 0.55 \\
\hline Callisthene major Mart. & SI & $\mathrm{D}$ & 2 & 2 & 3868.82 & 0.20 & 0.38 & 1.47 & 2 & 2 & 4036.17 & 0.21 & 0.39 & 1.46 \\
\hline $\begin{array}{l}\text { Campomanesia velutina } \\
\text { (Cambess.) O.Berg }\end{array}$ & $\mathrm{P}$ & $\mathrm{S}$ & 2 & 2 & 113.66 & 0.20 & 0.38 & 0.04 & 2 & 2 & 120.48 & 0.21 & 0.39 & 0.04 \\
\hline $\begin{array}{l}\text { Casearia gossypiosperma } \\
\text { Briq. }\end{array}$ & SI & I & 2 & 2 & 94.40 & 0.20 & 0.38 & 0.04 & 1 & 1 & 25.78 & 0.11 & 0.19 & 0.01 \\
\hline Cupania vernalis Cambess. * & SI & $\mathrm{I}$ & 2 & 2 & 340.53 & 0.20 & 0.38 & 0.13 & - & - & - & - & - & - \\
\hline $\begin{array}{l}\text { Garcinia gardneriana } \\
\text { (Planch. \& Triana) Zappi }\end{array}$ & ST & $\mathrm{I}$ & 2 & 2 & 78.88 & 0.20 & 0.38 & 0.03 & 3 & 3 & 117.38 & 0.32 & 0.58 & 0.04 \\
\hline Inga laurina (Sw.) Willd. & SI & $\mathrm{D}$ & 2 & 2 & 334.62 & 0.20 & 0.38 & 0.13 & 2 & 2 & 358.10 & 0.21 & 0.39 & 0.13 \\
\hline $\begin{array}{l}\text { Luehea grandiflora Mart. } \\
\text { \& Zucc. }\end{array}$ & $\mathrm{P}$ & I & 2 & 2 & 383.82 & 0.20 & 0.38 & 0.15 & 2 & 2 & 332.71 & 0.21 & 0.39 & 0.12 \\
\hline $\begin{array}{l}\text { Maclura tinctoria (L.) } \\
\text { D.Don ex Steud. }\end{array}$ & SI & $\mathrm{D}$ & 2 & 2 & 238.18 & 0.20 & 0.38 & 0.09 & 1 & 1 & 227.77 & 0.11 & 0.19 & 0.08 \\
\hline Maytenus floribunda Reissek & $\mathrm{ST}$ & $\mathrm{S}$ & 2 & 1 & 487.01 & 0.20 & 0.19 & 0.18 & 2 & 1 & 497.60 & 0.21 & 0.19 & 0.18 \\
\hline $\begin{array}{l}\text { Nectandra megapotamica } \\
\text { (Spreng.) Mez * }\end{array}$ & SI & $\mathrm{D}$ & 2 & 2 & 904.10 & 0.20 & 0.38 & 0.34 & - & - & - & - & - & - \\
\hline Sweetia fruticosa Spreng. & SI & $\mathrm{D}$ & 2 & 2 & 2545.37 & 0.20 & 0.38 & 0.97 & 3 & 3 & 2616.98 & 0.32 & 0.58 & 0.95 \\
\hline Trichilia catigua A. Juss. & SI & $\mathrm{S}$ & 2 & 2 & 55.96 & 0.20 & 0.38 & 0.02 & 1 & 1 & 38.52 & 0.11 & 0.19 & 0.01 \\
\hline Trichilia elegans A.Juss. & ST & $\mathrm{S}$ & 2 & 1 & 38.28 & 0.20 & 0.19 & 0.01 & 1 & 1 & 17.90 & 0.11 & 0.19 & 0.01 \\
\hline $\begin{array}{l}\text { Agonandra brasiliensis } \\
\text { Miers ex Benth. \& Hook.f. }\end{array}$ & ST & I & 1 & 1 & 232.05 & 0.10 & 0.19 & 0.09 & 1 & 1 & 258.55 & 0.11 & 0.19 & 0.09 \\
\hline $\begin{array}{l}\text { Alchornea glandulosa } \\
\text { Poepp. \& Endl. }\end{array}$ & $\mathrm{P}$ & $\mathrm{D}$ & 1 & 1 & 273.91 & 0.10 & 0.19 & 0.10 & 1 & 1 & 326.61 & 0.11 & 0.19 & 0.12 \\
\hline $\begin{array}{l}\text { Aspidosperma spruceanum } \\
\text { Benth. ex Müll.Arg. }\end{array}$ & ST & $\mathrm{D}$ & 1 & 1 & 35.09 & 0.10 & 0.19 & 0.01 & 1 & 1 & 38.52 & 0.11 & 0.19 & 0.01 \\
\hline
\end{tabular}


Tabela 1. Continuação

\begin{tabular}{|c|c|c|c|c|c|c|c|c|c|c|c|c|c|c|}
\hline \multirow{2}{*}{$\begin{array}{l}\text { Anos } \\
\text { Espécies }\end{array}$} & \multirow[b]{2}{*}{ GS } & \multirow[b]{2}{*}{ EV } & \multicolumn{6}{|c|}{2006} & \multicolumn{6}{|c|}{2011} \\
\hline & & & DA & FA & $\mathrm{AB}$ & DR & FR & DoR & DA & FA & $\mathrm{AB}$ & DR & FR & DoR \\
\hline $\begin{array}{l}\text { Aspidosperma subincanum } \\
\text { Mart. ex A.DC. }\end{array}$ & SI & $\mathrm{I}$ & 1 & 1 & 215.18 & 0.10 & 0.19 & 0.08 & 1 & 1 & 215.18 & 0.11 & 0.19 & 0.08 \\
\hline $\begin{array}{l}\text { Bauhinia rufa (Bong.) } \\
\text { Steud. * }\end{array}$ & SI & $\mathrm{S}$ & 1 & 1 & 43.95 & 0.10 & 0.19 & 0.02 & - & - & - & - & - & - \\
\hline Bauhinia ungulata L. * & $\mathrm{SI}$ & $\mathrm{S}$ & 1 & 1 & 76.47 & 0.10 & 0.19 & 0.03 & - & - & - & - & - & - \\
\hline $\begin{array}{l}\text { Calyptranthes clusiifolia } \\
\text { O.Berg }\end{array}$ & SI & $\mathrm{I}$ & 1 & 1 & 147.14 & 0.10 & 0.19 & 0.06 & 1 & 1 & 161.14 & 0.11 & 0.19 & 0.06 \\
\hline $\begin{array}{l}\text { Cardiopetalum calophyllum } \\
\text { Schltdl. * }\end{array}$ & SI & $\mathrm{S}$ & 1 & 1 & 31.83 & 0.10 & 0.19 & 0.01 & - & - & - & - & - & - \\
\hline $\begin{array}{l}\text { Cassia ferruginea (Schrad.) } \\
\text { Schrad. ex DC. }\end{array}$ & $\mathrm{SI}$ & $\mathrm{D}$ & 1 & 1 & 2370.00 & 0.10 & 0.19 & 0.90 & 1 & 1 & 3227.36 & 0.11 & 0.19 & 1.17 \\
\hline $\begin{array}{l}\text { Cecropia pachystachya } \\
\text { Trécul }\end{array}$ & $\mathrm{P}$ & $\mathrm{D}$ & 1 & 1 & 127.32 & 0.10 & 0.19 & 0.05 & 1 & 1 & 161.14 & 0.11 & 0.19 & 0.06 \\
\hline Celtis iguanaea (Jacq.) Sarg. & $\mathrm{P}$ & $\mathrm{S}$ & 1 & 1 & 64.64 & 0.10 & 0.19 & 0.02 & 2 & 2 & 152.87 & 0.21 & 0.39 & 0.06 \\
\hline Eugenia florida DC. * & SI & $\mathrm{I}$ & 1 & 1 & 81.49 & 0.10 & 0.19 & 0.03 & - & - & - & - & - & - \\
\hline $\begin{array}{l}\text { Eugenia ligustrina (Sw.) } \\
\text { Willd. }\end{array}$ & ST & $\mathrm{S}$ & 1 & 1 & 31.83 & 0.10 & 0.19 & 0.01 & 1 & 1 & 38.52 & 0.11 & 0.19 & 0.01 \\
\hline Ficus guaranitica Chodat & SI & $\mathrm{D}$ & 1 & 1 & 24.37 & 0.10 & 0.19 & 0.01 & 1 & 1 & 24.37 & 0.11 & 0.19 & 0.01 \\
\hline Ficus sp. & SI & $\mathrm{D}$ & 1 & 1 & 1233.47 & 0.10 & 0.19 & 0.47 & 1 & 1 & 1243.40 & 0.11 & 0.19 & 0.45 \\
\hline $\begin{array}{l}\text { Guarea guidonia (L.) } \\
\text { Sleumer }\end{array}$ & ST & I & 1 & 1 & 53.79 & 0.10 & 0.19 & 0.02 & 1 & 1 & 53.79 & 0.11 & 0.19 & 0.02 \\
\hline Guazuma ulmifolia Lam. & $\mathrm{P}$ & I & 1 & 1 & 453.61 & 0.10 & 0.19 & 0.17 & 1 & 1 & 453.61 & 0.11 & 0.19 & 0.16 \\
\hline $\begin{array}{l}\text { Guettarda viburnoides } \\
\text { Cham. \& Schltdl. * }\end{array}$ & SI & I & 1 & 1 & 38.52 & 0.10 & 0.19 & 0.01 & - & - & - & - & - & - \\
\hline $\begin{array}{l}\text { Lacistema aggregatum } \\
\text { (P.J.Bergius) Rusby }\end{array}$ & $\mathrm{P}$ & $\mathrm{S}$ & 1 & 1 & 21.66 & 0.10 & 0.19 & 0.01 & 1 & 1 & 21.66 & 0.11 & 0.19 & 0.01 \\
\hline $\begin{array}{l}\text { Machaerium hirtum (Vell.) } \\
\text { Stellfeld }\end{array}$ & $\mathrm{P}$ & I & 1 & 1 & 1461.06 & 0.10 & 0.19 & 0.55 & 1 & 1 & 1461.06 & 0.11 & 0.19 & 0.53 \\
\hline Machaerium opacum Vogel & $\mathrm{P}$ & I & 1 & 1 & 658.98 & 0.10 & 0.19 & 0.25 & 1 & 1 & 710.65 & 0.11 & 0.19 & 0.26 \\
\hline $\begin{array}{l}\text { Peltophorum dubium } \\
\text { (Spreng.) Taub. }\end{array}$ & $\mathrm{P}$ & $\mathrm{D}$ & 1 & 1 & 24.37 & 0.10 & 0.19 & 0.01 & 1 & 1 & 62.39 & 0.11 & 0.19 & 0.02 \\
\hline Psidium rufum Mart. ex DC. & SI & $\mathrm{S}$ & 1 & 1 & 27.24 & 0.10 & 0.19 & 0.01 & 1 & 1 & 31.83 & 0.11 & 0.19 & 0.01 \\
\hline Qualea multiflora Mart. & ST & $\mathrm{D}$ & 1 & 1 & 1223.58 & 0.10 & 0.19 & 0.46 & 1 & 1 & 1283.51 & 0.11 & 0.19 & 0.46 \\
\hline Xylopia sericea A.St.-Hil. & $\mathrm{P}$ & $\mathrm{D}$ & 1 & 1 & 66.92 & 0.10 & 0.19 & 0.03 & 1 & 1 & 76.47 & 0.11 & 0.19 & 0.03 \\
\hline $\begin{array}{l}\text { Campomanesia guazumifolia } \\
\text { (Cambess.) O.Berg * }\end{array}$ & SI & $\mathrm{S}$ & - & - & - & - & - & - & 1 & 1 & 23.00 & 0.11 & 0.19 & 0.01 \\
\hline Xylopia brasiliensis Spreng.* & $\mathrm{ST}$ & $\mathrm{D}$ & - & - & - & - & - & - & 1 & 1 & 23.00 & 0.11 & 0.19 & 0.01 \\
\hline $\begin{array}{l}\text { Zanthoxylum rhoifolium } \\
\text { Lam. * }\end{array}$ & SI & D & - & - & - & - & - & - & 1 & 1 & 64.64 & 0.11 & 0.19 & 0.02 \\
\hline Totais & & & 976 & 526 & 263356.9 & 100 & 100 & 100 & 934 & 517 & 276142.0 & 100 & 100 & 100 \\
\hline
\end{tabular}

Tabela 2. Teste de Friedman para taxas de dinâmica em diferentes estratos e em diferentes grupos sucessionais em floresta estacional semidecidual. Letras representam o resultado do teste a posteriori de Bonferroni, onde $\mathrm{a}>\mathrm{b}>\mathrm{c}$. Graus de liberdade para todos os testes foram 2 . Mort. $=$ Taxa de mortalidade, Recr. $=$ Taxa de recrutamento, Decr. $=$ Taxa de decremento, Incr. $=$ Taxa de incremento, Dos. $=$ Dossel, Int. $=$ Intermediário, Sub. = Sub-bosque, Fried. $=$ Valor do teste de Friedman, $\mathrm{P}=$ Pioneiras, $\mathrm{SI}=$ Secundárias iniciais, $\mathrm{ST}=\mathrm{Secundárias} \mathrm{tardias}$.

\begin{tabular}{|c|c|c|c|c|c|c|c|c|c|c|}
\hline Taxa & Dos. & Int. & Sub. & Fried. & $\mathrm{p}$ & $\mathrm{P}$ & SI & ST & Fried. & $\mathrm{p}$ \\
\hline Mort. & $1.35^{\mathrm{c}}$ & $2.73^{b}$ & $7.70^{\mathrm{a}}$ & 20.52 & $<0.001$ & $2.03^{b}$ & $4.39^{\mathrm{a}}$ & $1.42^{b}$ & 14.87 & $<0.001$ \\
\hline Recr. & $1.23^{\mathrm{b}}$ & $1.92^{\mathrm{b}}$ & $5.13^{\mathrm{a}}$ & 10.33 & 0.006 & $0.90^{\mathrm{b}}$ & $3.06^{\mathrm{a}}$ & $1.26^{\mathrm{b}}$ & 19.32 & $<0.001$ \\
\hline Decr. & $1.01^{\mathrm{b}}$ & $1.89^{\mathrm{b}}$ & $5.40^{\mathrm{a}}$ & 16.42 & $<0.001$ & $1.92^{\mathrm{a}}$ & $1.63^{b}$ & $1.00^{\mathrm{b}}$ & 12.65 & 0.002 \\
\hline Incr. & $2.02^{\mathrm{c}}$ & $2.96^{\mathrm{b}}$ & $4.67^{\mathrm{a}}$ & 14.64 & $<0.001$ & $2.71^{\mathrm{a}}$ & $2.50^{\mathrm{a}}$ & $1.99^{\mathrm{a}}$ & 2.24 & 0.32 \\
\hline
\end{tabular}


dossel apresentou as menores taxas de dinâmica, indicando uma maior estabilidade e refletindo a dicotomia nas estratégias de vida entre as espécies de sub-bosque e de dossel.

A dinâmica mais acelerada no sub-bosque pode ser resultado do ciclo de vida mais curto das espécies desse estrato em relação à maioria das espécies que ocupam os estratos superiores (Lieberman et al. 1985, Manokaran \& Kochummen 1987). Com base na teoria de história de vida, espécies florestais de menor estatura adulta podem ser consideradas R-estrategistas, apresentando menor longevidade e reprodução precoce, enquanto espécies de maior estatura são K-estrategistas, alocando recursos para crescimento a fim de atingirem o dossel e a idade reprodutiva (Pianka 1970, Poorter et al. 2008). Além disso, espécies de sub-bosque tendem a apresentar maiores taxas de mortalidade e decremento porque estão mais vulneráveis aos danos causados pela queda de galhos e de grandes árvores (Poorter et al. 2006), têm menores raízes e estão mais sujeitas à competição por água (Ibáñez et al. 2007), e estão mais próximas do período de senescência considerandose o critério de inclusão amostrado (CAP $\geq 15 \mathrm{~cm}$ ), já que muitas das espécies de sub-bosque, ao atingirem o critério de inclusão, são compostas por indivíduos já adultos.

Embora alguns estudos sugiram que a luz não é um recurso limitante para as espécies em florestas secas, já que possuem dossel mais baixo e aberto comparado a florestas tropicais úmidas (Lebrija-Trejos et al. 2008, Lohbeck et al. 2013), outros apontam que, para os estratos inferiores, a luz continua sendo um filtro ecológico importante para o desenvolvimento das espécies de florestas secas (Prado Junior et al. 2016). Dessa forma, a limitação por luz pode ser outro fator que explica a maior mortalidade das espécies do sub-bosque comparado aos outros estratos.

As maiores taxas de recrutamento das espécies do sub-bosque podem ser resultado da alocação precoce de recursos para a reprodução, quando comparadas às espécies dos estratos superiores (Thomas 2011), o que se refletiu em maiores taxas de incremento neste estrato. Os recrutas representaram 38\% do incremento absoluto em área basal (comparado com 16\% do estrato intermediário e 3\% do dossel). Apesar disso, o valor absoluto de incremento em área basal do sub-bosque representou apenas $5 \%$ do valor total da comunidade.

O estrato intermediário apresentou taxas intermediárias entre as do sub-bosque e dossel, o que pode indicar uma grande variedade de estratégias e um contínuo de ocupação espacial entre o dossel e o sub-bosque, assim como condições intermediárias quanto à luminosidade (Vale et al. 2009).

A menor taxa líquida de número de indivíduos (recrutamento menos mortalidade) foi registrada no subbosque, responsável por $66 \%$ da diminuição na densidade arbórea observada na comunidade. Com o avanço sucessional, espécies de menor porte tendem a reduzir sua importância na comunidade arbórea (Machado \& Oliveira-
Filho 2010). O mesmo ocorreu com as taxas líquidas de área basal (incremento menos decremento), negativa para o sub-bosque e positiva para os demais estratos. Assim, o aumento em área basal e consequente o ganho em biomassa das florestas com o avanço sucessional está concentrado nos estratos superiores da floresta. Espécies de estratos superiores interceptam uma maior quantidade de luz e podem se sobrepor a indivíduos vizinhos, apresentando maior crescimento (Poorter et al. 2008). Lohbeck et al. (2013) indicam que, com o avanço sucessional em florestas secas, pode haver uma inversão nos recursos que limitam estas florestas. Em estágios iniciais, quando o dossel é mais baixo e esparso e a incidência luminosa e exposição à desidratação são maiores, a água é o principal recurso limitante. Posteriormente, com o avanço sucessional, o aumento do dossel cria condições microclimáticas menos suscetíveis ao estresse hídrico, e a luz passa a ser um fator limitante para o desenvolvimento das plantas.

\section{Dinâmica entre os grupos sucessionais}

As espécies pioneiras, secundárias iniciais e secundárias tardias representaram, respectivamente, 7\%, 54\% e 39\% da média dos indivíduos amostrados nos dois tempos (Tab. 1), valores similares aos observados em outras florestas estacionais semideciduais conservadas na região (Prado Júnior et al. 2010, 2012, Lopes et al. 2014). Devido ao avanço sucessional da floresta, o grupo das pioneiras (P) apresentou as menores taxas de recrutamento (Tab. 2), o que pode indicar que o seu recrutamento esteja restrito às clareiras formadas por quedas de galhos ou de árvores, devido à ausência de impactos antrópicos diretos na floresta (Tabarelli \& Mantovani 1997).

O grupo das espécies secundárias iniciais (SI) apresentou as maiores taxas de recrutamento e mortalidade. Por tolerarem certo grau de sombreamento, em estágios mais avançados da sucessão acabam apresentando maior recrutamento do que espécies pioneiras, mas ao mesmo tempo têm um ciclo de vida mais curto do que as espécies secundárias tardias, apresentando maior mortalidade (Kohler et al. 2000). Dessa forma, as características deste grupo podem ser consideradas uma transição entre aquelas de pioneiras e secundárias tardias (Lopes et al. 2014).

O grupo das espécies secundárias tardias (ST) apresentou as menores taxas tanto de incremento quanto de mortalidade. Essas espécies podem persistir por um longo tempo sob condições de maior sombreamento do subbosque e podem alocar mais recursos para o crescimento da área de copa do que em diâmetro (Prado Junior et al. 2016).

As maiores taxas de decremento e incremento foram observadas para as $\mathrm{P}$, e as menores para as ST, mas que por sua vez tiveram as maiores taxas líquidas de densidade e de área basal. A redução na densidade arbórea e o aumento na área basal refletem uma relação de compromisso entre crescimento e sobrevivência (Poorter et al. 2008), evidenciada pelo contraste do crescimento rápido das $\mathrm{P}$ para que possam atingir os estratos superiores com maior luminosidade mas com menor longevidade, e do 
crescimento mais lento mas com maior longevidade das ST (Wright et al. 2010).

Os resultados apresentados neste estudo indicam que, embora seja um fragmento pequeno, imerso em uma matriz antropizada, a floresta estacional semidecidual da Fazenda do Glória está avançando no processo sucessional. A ausência de impactos diretos como corte seletivo e entrada de gado na floresta favorecem este avanço sucessional (Lopes et al. 2013). Futuros estudos avaliando a dinâmica de outros aspectos ecológicos (como síndromes de dispersão) e das populações dominantes são necessários para investigar processos mais complexos como a homogeneização em fragmentos florestais isolados e a consequente redução na riqueza de espécies de dispersão zoocórica, especialmente aquelas associadas aos frugívoros de grande porte.

\section{AGRADECIMENTOS}

Ao Programa de Pós-Graduação em Biologia Vegetal da Universidade Federal de Uberlândia pelo apoio. À Prof. Dra. Ana Paula de Oliveira e à Dra. Carolina Silvério Arantes pelas contribuições. À Coordenação de Aperfeiçoamento de Pessoal de Nível Superior (CAPES), ao Conselho Nacional de Desenvolvimento Científico e Tecnológico (CNPQ) e ao Programa de Pesquisa Ecológica de Longa Duração (PELD) pelo apoio financeiro. Ao Programa de Concessão de Bolsa de Incentivo ao Pesquisador (BIP/UEG).

\section{REFERÊNCIAS}

Alvares, C.A., Stape, J.L., Sentelhas, P.C., Gonçalves, J.L.M. \& Sparovek, G. 2013. Köppen's climate classification map for Brazil. Meteorologische Zeitschrift 22(6): 711-728.

Angiosperm Phylogeny Group IV. 2016. An update of the Angiosperm Phylogeny Group classification for the orders and families of flowering plants: APG IV. Botanical Journal of the Linnean Society 181:1-20.

Appolinário, V., Oliveira-Filho, A.T. \& Guilherme, F.A.G. 2005. Tree population and community dynamics in a Brazilian tropical semideciduous Forest. Revista Brasileira de Botânica 28(2):347-360.

Brower, J.E; Zar, J.H.; Von Ende, C.N. 1997. Field and laboratory methods for general Ecology. 4th ed. WCB McGraw-Hill, New York. 273p.

Budowski, G. 1965. Distribution of tropical American forest species in a light of successional processes. Turrialba 15:40-42.

Chagas, R.K., Oliveira-Filho, A.T., Berg, E. \& Scolforo, J.R.S. 2001. Dinâmica de populações arbóreas em um fragmento de floresta estacional semidecidual em Lavras, Minas Gerais. Revista Árvore 25(1):39-57.

Chazdon, R.L. 2008. Chance and determinism in tropical forest succession. In Tropical forest community ecology. (W.P. Carson \& S.A. Schnitzer, eds.). Blackwell Publishing Ltd. Chichester, p. 384-408.

Chazdon, R.L. 2012. Regeneração de florestas tropicais. Boletim do Museu Paraense Emílio Goeldi. Ciências Naturais 7(3):195-218.

Chazdon, R.L., Letcher, S.G., Van Breugel, M., Martínez-Ramos, M., Bongers, F. \& Finegan, B. 2007. Rates of change in tree communities of secondary Neotropical forests following major disturbances. Philosophical Transactions of the Royal Society B: Biological Sciences 362:273-289.

Delcamp, M., Gourlet-Fleury, S., Flores, O. \& Garnier, E. 2008. Can functional classification of tropical trees predict population dynamics after disturbance? Journal of Vegetation Science 19:209-220.

Dias Neto, O.C., Schiavini, I., Lopes, S.F., Vale, V.S., Gusson, A.E. \& Oliveira, A.P. 2009. Estrutura fitossociológica e grupos ecológicos em fragmento de floresta estacional semidecidual, Uberaba, Minas Gerais, Brasil. Rodriguésia 60(4):1087-1100.
Durigan, G., Siqueira, M.F. \& Franco, G.A.D.C. 2002. A vegetação do Estado de São Paulo. In Biodiversidade, Conservação e Uso Sustentável da Flora do Brasil (L. Araújo, A.N. Moura, E.S.B. Sampaio, L.M.S.G. Gestinari \& J.M.T. Carneiro, eds.). Imprensa Universitária, Universidade Federal Rural de Pernambuco, Recife, p. 53-54.

Flora do Brasil 2020 em construção. Jardim Botânico do Rio de Janeiro. Disponível em: http://floradobrasil.jbrj.gov.br/ . Acessado em 09.08.2017.

Gandolfi, S., Leitão-Filho, H.F. \& Bezerra, C.L.F. 1995. Levantamento florístico e caráter sucessional das espécies arbustivo-arbóreas de uma floresta mesófila semidecídua no município de Guarulhos, SP. Revista Brasileira de Biologia 55:753-767.

Gomes, E.P.C., Mantovani, W. \& Kageyama, P.Y. 2003. Mortality and recruitment of trees in a secondary montane rainforest in southeastern Brazil. Brazilian Journal of Biology 63:47-60.

Haridasan, M. \& Araújo, G.M. 2005. Perfil nutricional de espécies lenhosas de duas florestas semidecíduas em Uberlândia, MG. Revista Brasileira de Botânica 28:295-303.

Higuchi, P., Oliveira-Filho, A.T., Silva, A.C., Machado, E.L.M., Santos, R.M. \& Pifano, D.S. 2008. Dinâmica da comunidade arbórea em um fragmento de floresta estacional semidecidual montana em Lavras, Minas Gerais, em diferentes classes de solos. Revista Árvore 32:417426.

Ibáñez, I., Clark, J.S., LaDeau, S. \& Lambers, J.H.R. 2007. Exploiting temporal variability to understand tree recruitment response to climate change. Ecological Monographs 77(2):163-177.

Kohler, P., Ditzer, T. \& Huth A. 2000. Concepts for the aggregation of tropical tree species into functional types and the application to Sabah's lowland rain forests. Journal of Tropical Ecology 16:591-602.

Laurance, W.F., Nascimento, H.E.M., Laurance, S.G., Andrade, A., Ribeiro, J.E.L.S., Giraldo, J.P., Lovejoy, T.E., Condit, R., Chave, J., Harms, K.E. \& D'Angelo, S. 2006 Rapid decay of tree-community composition in Amazonian forest fragments. Proceedings of the National Academy of Sciences 103(50):19010- 19014.

Lebrija-Trejos, E., Bongers, F., Perez-Garcia, E.A. \& Meave, J.A. 2008. Successional change and resilience of a very dry tropical deciduous forest following shifting agriculture. Biotropica 40:422-431

Lieberman, D., Lieberman, M., Hartshorn, G. \& Peralta, R. 1985. Growth rates and age-size relationships of tropical wet forest trees in CostaRica. Journal of Tropical Ecology 1:97-109.

Lohbeck, M., Poorter, L., Lebrija-Trejos, E., Martínez-Ramos, M., Meave, J.A., Paz, H., Perez-Garcia, E.A., Romero-Perez, I.E., Tauro, A. \& Bongers, F. 2013. Successional changes in functional composition contrast for dry and wet tropical forest. Ecology 94(6):1211-1216.

Lopes, S.F., Prado Júnior, J.A., Vale, V.S. \& Schiavini, I. 2013. Impactos ambientais antrópicos como modificadores da estrutura e funcionalidade de florestas estacionais semideciduais no Triângulo Mineiro, Brasil. Caminhos de Geografia Uberlândia 14(47):233-242.

Lopes, S.F., Schiavini, I., Prado Júnior, J.A., Gusson, A.E., Souza Neto, A.R., Vale, V.S. \& Dias Neto, O.C. 2011. Caracterização ecológica e distribuição diamérica da vegetação arbórea de um remanescente de floresta estacional semidecidual, na Fazenda Experimental do Glória, Uberlândia, MG. Bioscience Journal 27(2):322-335.

Lopes, S.F., Vale, V.S., Schiavini, I., Júnior, J.A.P., Oliveira, A.P., \& Arantes, C.S. 2014. Canopy stratification in tropical seasonal forests: how the functional traits of community change among the layers. Bioscience Journal 30(5):1551-1562.

Machado, E.L.M. \& Oliveira-Filho, A.T. 2010. Spatial patterns of tree community dynamics are detectable in a small (4 ha) and disturbed fragment of the Brazilian Atlantic forest. Acta Botanica Brasilica 24(1):250-261.

Manokaran, N. \& Kochummen, K.M. 1987. Recruitment, growth and mortality of tree species in a lowland Dipterocarp Forest in peninsular Malaysia. Journal of Tropical Ecology 3:315-330.

Meira-Neto, J.A.A., Martins, F.R. \& Souza, A.L. 2005. Influência da cobertura e do solo na composição florística do sub-bosque em uma floresta estacional semidecidual em Viçosa, MG, Brasil. Acta Botanica Brasilica 19(3):473-486.

Mueller-Dombois, D. \& Ellenberg, H. 1974. Aims and methods of vegetation ecology. John Wiley \& Sons, New York. 547p. 
Nunes, Y.R.F., Mendonça, A.V.R., Botzelli, L., Machado, E.L.M. \& Oliveira Filho, A.T. 2003. Variações da fisionomia, diversidade e composição de guildas da comunidade arbórea em um fragmento de floresta semidecidual em Lavras, MG. Acta Botanica Brasilica 17:213-229.

Odum, E.P. 2001. Fundamentos de ecologia. Fundação Calouste Gulbenkian, Lisboa. 927p.

Oliveira, A.P., Schiavini, I., Vale, V.S., Lopes, S.F., Arantes, C.S., Gusson, A.E., Prado Júnior, J.A. \& Dias-Neto, O.C. 2014. Mortality, recruitment and growth of the tree communities in three forest formations at the Panga Ecological Station over ten years (19972007). Acta Botanica Brasilica 28(2):234-248.

Oliveira-Filho, A.T., Carvalho, W.A.C., Machado, E.L.M., Higuchi, P., Appolinário, V., Castro, G.C., Silva, A.C., Santos, R.M., Borges, L.F., Corrêa, B.S. \& Alves, J.M. 2007. Dinâmica da comunidade e populações arbóreas da borda e interior de um remanescente florestal na Serra da Mantiqueira, Minas Gerais, em um intervalo de cinco anos (1999-2004). Revista Brasileira de Botânica 30(1):149-161.

Oliveira-Filho, A.T., Mello, J.M. \& Scolforo, J.R.S. 1997. Effects of past disturbance and edges on tree community structure and dynamics within a fragment of tropical semideciduous forest in South-eastern Brazil over a five-year period (1987-1992). Plant Ecology 131(1):4566.

Parthasarathy, N. 1999. Tree diversity and distribution in undisturbed and human-impacted sites of tropical wet evergreen forest in southern Western Ghats, India. Biodiversity and Conservation 8(4):1365-1381.

Pianka, E.R. 1970. On R- and K-selection. The American Naturalist 104:592-597.

Poorter, L., Bongers, L. \& Bongers, F. 2006. Architecture of 54 moistforest tree species: Traits, trade-offs, and functional groups. Ecology 87: 1289-1301.

Poorter, L., Wright, S.J., Paz, H., Ackerly, D.D., Condit, R., IbarraManríquez, G., Harms, K.E., Licona, J.C., Martínez-Ramos, M., Mazer, S.J., Muller-Landau, H.C., Peña-Claros, M., Webb, C.O. \& Wright, I.J. 2008. Are functional traits good predictors of demographic rates? Evidence from five neotropical forests. Ecology 89:1908-1920.

Prado Júnior, J.A, Lopes, S.F., Schiavini, I., Vale V.S., Oliveira, A.P., Gusson, A.E., Dias Neto, O.C. \& Stein, M. 2012. Fitossociologia, caracterização sucessional e síndromes de dispersão da comunidade arbórea de remanescente urbano de Floresta Estacional Semidecidual em Monte Carmelo, Minas Gerais. Rodriguésia 63(3):489-499.

Prado Junior, J.A., Schiavini, I., Vale, V.S., Arantes, C.S., Sande, M.T.,
Lohbeck, M. \& Poorter, L. 2016. Conservative species drive biomass productivity in tropical dry forests. Journal of Ecology 104:817-827.

Prado Júnior, J.A., Vale, V.S., Oliveira, A., Gusson, A.E., Dias Neto, O.C., Lopes, S.F. \& Schiavini, I. 2010. Estrutura da comunidade arbórea em um fragmento de floresta estacional semidecidual localizada na reserva legal da Fazenda Irara, Uberlândia, MG. Bioscience Journal 26(4):638-647.

Prado Júnior, J.A., Vale, V.S., Lopes, S.F, Arantes, C.S., Oliveira, A.P. \& Schiavini, I. 2014. Impacts of disturbance intensity in functional traits patterns in understories of seasonal forests. Bioscience Journal 30(5):901-911

Rodrigues-Souza, J.R., Prado Júnior, J.A., Vale, V.S., Schiavini, I., Oliveira, A. P., \& Arantes, C.S. 2015. Secondary forest expansion over a savanna domain at an ecological reserve in the Southeastern Brazil after 15 years of monitoring. Brazilian Journal of Botany 38(2):311-322.

Sheil, D., Burslem, D.F.R.P. \& Alder, D. 1995. The interpretation and misinterpretation of mortality rate measures. Journal of Ecology 83:331-333.

Sheil, D., Jennings, S. \& Savill, P. 2000. Long-term permanent plot observations of vegetation dynamics in Bundongo, a Ugandan rain forest. Journal of Tropical Ecology 16(6):675-800.

Shepherd, G.J., 2009. FITOPAC 2.1. Universidade Estadual de Campinas, Campinas.

Tabarelli, M. \& Mantovani, W. 1997. Colonização de clareiras naturais na floresta atlântica no sudeste do Brasil. Revista Brasileira de Botânica 20(1):57-66.

Thomas, S.C. 2011. Age-related changes in tree growth and functional biology: the role of reproduction. In Size-and age-related changes in tree structure and function. (F.C. Meinzer, B. Lachenbruch \& T.E. Dawson, eds.) Springer Netherlands, Dordrecht, p. 33-64.

Vale, V.S., Schiavini, I., Lopes, S.F., Dias Neto, O.C., Oliveira, A.P. \& Gusson, A.E. 2009. Composição florística e estrutura do componente arbóreo de um remanescente primário de floresta estacional semidecidual em Araguari, Minas Gerais, Brasil. Hoehnea 36(3):417429.

Whitmore, T.C. 1989. Canopy gaps and the two major groups of forest trees. Ecology 70:536 - 538.

Wright, S.J., Kitajima, K., Kraft, N.J., Reich, P.B., Wright, I.J., Bunker, D.E., Condit, R., Dalling, J.W., Davies, S.J., Diaz, S., Engelbrecht, B.M.J., Harms, K.E., Hubbell, S.P., Marks, C.O., Ruiz-Jaen, M.C., Salvador, C.M. \& Zanne, A.E. 2010. Functional traits and the growthmortality trade-off in tropical trees. Ecology 91(12):3664-3674. 